\title{
Ferramenta de Apoio ao Planejamento Educacional
}

\author{
Dirceu Antonio Maraschin $\mathbf{J r}^{1}$, \\ Fabiane Beletti da Silva ${ }^{2}$, \\ Tiago Thompsen Primo ${ }^{1}$, \\ Raymundo Carlos Machado Ferreira Filho ${ }^{3}$, \\ Lucas Mendes Tortelli ${ }^{1}$, \\ Margarete Hirdes Antunes ${ }^{3}$, \\ Thiago Medeiros Barros ${ }^{4}$, \\ Tatiana Lebedeff ${ }^{2}$ \\ ${ }^{1}$ CDTec - Universidade Federal de Pelotas (UFPel) - Pelotas - RS - Brasil \\ \{dmaraschin, tiago.primo, lmtortelli\}@inf.ufpel.edu.br \\ ${ }^{2}$ Centro de Letas e Comunicação - Universidade Federal de Pelotas (UFPel) \\ \{fabiclmd, tblebedeff\}@gmail.com \\ ${ }^{3}$ Instituto Federal de Educação, Ciência e Tecnologia Sul-riograndense \\ Campus Visconde da Graça - Pelotas - RS - Brasil \\ raymundofilho@cavg.ifsul.edu.br, npte.margarete@gmail.com \\ ${ }^{4}$ Instituto Federal do Rio Grande do Norte - Natal - RN - Brasil \\ thiago.medeiroseifrn.edu.br
}

\begin{abstract}
This article intends to contribute by making visible the solution found for one of the biggest challenges in educational planning and the development of educational resources, teaching methods and strategies, being related to the support, storage and recovery of the memory associated with its process elaboration. In this work, as a way to face this challenge, we propose a tool that implements the ADDIE model based on the question-answer technique and skills of the Educational Designer. We present a set of technological components to store, process, retrieve and recommend content, as well as suggestions for practices to support the preparation of educational project.
\end{abstract}

Resumo. O presente artigo pretende contribuir visibilizando a solução encontrada para um dos maiores desafios no planejamento educacional e no desenvolvimento de recursos educacionais, métodos e estratégias de ensino, estando relacionado ao suporte, armazenamento e recuperação da memória associada ao seu processo de elaboração. Neste trabalho, como enfrentamento ao referido desafio, propomos uma ferramenta que implementa o modelo ADDIE com base na técnica de pergunta-resposta e habilidades do Designer Educacional. Apresentamos um conjunto de componentes tecnológicos para armazenar, processar, recuperar e recomendar conteúdos, bem como sugestões de práticas para subsidiar a elaboração do projeto educacional. 
IX Congresso Brasileiro de Informática na Educação (CBIE 2020)

Anais do XXXI Simpósio Brasileiro de Informática na Educação (SBIE 2020)

\section{Introdução}

O compartilhamento de informações é prática usual nos dias atuais. Este fato que pode ser constatado, de forma empírica, apenas observando o cotidiano das redes sociais, onde o processo de "compartilhar" está presente em boa parte dos espaços virtuais de convivência. Se pensarmos nos ambientes acadêmicos, o compartilhamento de informações deve ser realizado de maneira apropriada, pois a essência do que se compartilha não é somente informativa, mas sim de socialização de produções técnicas e intelectuais a partir de métodos científicos.

Considerando a produção técnico-científica para formação profissional, circunscrita à Rede de Institutos Federais, foi constatada a necessidade de compartilhamento dos recursos educacionais, métodos e estratégias de ensino desenvolvidos e validados pela Rede. A produção destes conteúdos envolve muitas trocas de informações visando a qualidade do material produzido. Neste ambiente surgiu o PROEDU, um repositório de recursos educacionais abertos que visa armazenar, disponibilizar e dar acesso à produção realizada nestes ambientes. Se considerarmos o fomento desta produção, entende-se como necessário o compartilhamento do conhecimento, dos recursos educacionais, métodos e estratégias que levaram a concepção deste conteúdo educacional.

Em síntese, o PROEDU materializa a memória da produção intelectual desenvolvida para uso em cursos de formação profissionalizante da Rede de Institutos Federais, onde, além de cumprir um papel social de disseminação do conhecimento, proporciona a otimização do trabalho das equipes de produção, no momento em que disponibiliza recursos educacionais, métodos e estratégias de ensino que podem ser adaptados e novamente socializados, atendendo a demanda pontual de cada oferta de cursos da Rede.

Neste sentido, observou-se a necessidade de realizar o registro formal da concepção destes produtos (por exemplo: recursos educacionais, métodos e estratégias de ensino e materiais relacionados que foram utilizados como norte no planejamento educacional). Uma das formas de conservar a memória das produções é através de um documento de referência denominado Projeto Instrucional ou Projeto Educacional, norteado por métodos de design educacional já consolidados, pois o registro desta memória de produção supostamente favorece a adaptação dos produtos por outros grupos ou instituições. A fim de atender a esta demanda, o PROEDU propõe a concepção e estruturação de uma ferramenta que fará o apoio ao desenvolvimento do documento de referência denominado Projeto Educacional (PE), amparado por um método de design educacional. O objetivo geral da presente pesquisa é: desenvolver uma solução de apoio à composição de Projetos Educacionais (PE) a partir de um método de Design Educacional (DE). Este desafio requer análise da experiência de usuário, sendo este processo apoiado no trabalho de (Zilse et al. 2016).

Para alcançar o objetivo indicado, propõe-se uma solução que implementa o modelo ADDIE para composição de PE. Nela estão previstos componentes tecnológicos que visam apoiar, facilitar e enriquecer o nível de detalhamento do $\mathrm{PE}$ a ser produzido com o uso da ferramenta.

Fazem parte dos desafios a elaboração de componentes tecnológicos que possibilitem adaptar um método de design educacional no desenvolvimento de projetos educacionais, para a Educação Profissional e Tecnológica considerando: 
- Registro da memória da concepção e desenvolvimento de recursos educacionais, métodos e estratégias de ensino, as teorias de aprendizagem que amparam o planejamento e as tomadas de decisões, facilitando a utilização e adaptação destes produtos pela comunidade acadêmica em geral.

- Utilizar um método de design educacional, nas bases de desenvolvimento de uma ferramenta de apoio, poderá reduzir as taxas de erro no momento da implantação ou adaptação dos produtos em cursos ou disciplinas.

- As técnicas de recomendação, incorporadas à ferramenta, poderão qualificar determinadas decisões metodológicas, acelerando e desonerando o trabalho do designer educacional.

- A ação sistemática de escrita dos projetos educacionais poderá auxiliar no desenvolvimento da cultura de registro formal da concepção de recursos educacionais, métodos e estratégias de ensino, pois trarão em seus registros o detalhamento necessário para utilização e adequação das produções efetuadas.

O trabalho está organizado da seguinte forma: na seção 3, apresenta-se a base teórica do trabalho, compreendendo conceitos do Design Educacional e o princípio do modelo ADDIE, apresentando suas fases, etapas e produtos gerados após cada etapa. Na seção 4, discursa-se sobre as etapas de análise e desenho da solução com base no modelo ADDIE, apresenta-se também a matriz de DE adotada no PROEDU. Após, na seção 5, apresenta-se a ferramenta de geração de projetos educacionais, contemplando a concepção da ideia de design da aplicação e sua composição tecnológica. Na seção 6 são dadas as considerações finais. Logo, encerra-se o trabalho apresentando as referência bibliográficas.

\section{Trabalhos Relacionados}

Como iniciativas semelhantes à proposta apresentada neste trabalho, pode-se citar aplicações que se propõem à criação de recursos educacionais compartilháveis e interativos: Hot Potatoes ${ }^{1}$, atualmente, na sétima versão é um pacote de seis aplicativos que permite a criação de diferentes tipos de exercícios para a internet (como palavras cruzadas, múltipla escolha, ordenação, completar lacunas, etc.), é um software livre, porém não de código aberto e sua versão Java está disponível para download, compatível com diferentes sistemas operacionais. eXeLearning ${ }^{2}$ é também software livre, open-source, que permite criar conteúdos no formato XHTML ou HTML5e páginas web, permitindo exportá-los em diferentes formatos para serem usados independentemente ou integrados a um sistema de gestão de aprendizagem, como por exemplo, o Moodle.

O modelo para design de atividades envolvendo aprendizagem criativa, desenvolvido pelo MIT Media Lab e amplamente utilizado pela Rede Brasileira de Aprendizagem Criativa ${ }^{3}$, inspirou alguns pontos na proposta presente neste trabalho, sobretudo ao considerar um espaço para listar fatores de "Inspiração" na composição do PE. Entretanto, estes ambientes se distanciam da proposta de geração de projetos educacionais, pois não implementam nenhuma metodologia consolidada, tampouco estão direcionadas ao desenvolvimento de recursos educacionais ou parte do processo de composição.

\footnotetext{
${ }^{1}$ hotpot.uvic.ca/

${ }^{2}$ exelearning.net/en/

${ }^{3}$ aprendizagemcriativa.org
} 
IX Congresso Brasileiro de Informática na Educação (CBIE 2020)

Anais do XXXI Simpósio Brasileiro de Informática na Educação (SBIE 2020)

\section{Fundamentos Conceituais}

Quando uma instituição se propõe a planejar recursos educacionais, métodos e estratégias de ensino, o objetivo fim é proporcionar a aprendizagem dos estudantes para os quais se destinam, bem como a formação destes estudantes enquanto seres sociais (Libâneo 1992). Qualquer que seja a modalidade de ensino considerada, para que estudantes vivenciem os processos de aprendizagem de forma eficiente, é prudente analisar o contexto no qual as formações serão inseridas, o público-alvo ao qual se destinam e os objetivos que se pretende alcançar com os processos de aprendizagem. Zabala (Zabala 2015) insiste que tudo que se realiza no processo educativo interfere na formação dos alunos.

A maneira de organizar a aula, o tipo de incentivos, as expectativas que depositamos, os materiais que utilizamos, cada uma destas decisões veicula determinadas experiências educativas. Para que esta situação ideal ocorra na prática, o planejamento dos recursos educacionais, métodos e estratégias de ensino configura-se como parte fundamental neste processo.

Considerando a importância do método científico para desenvolvimento da sociedade, independente da área de conhecimento, embasar o planejamento educacional em métodos já consolidados, como metodologias utilizadas na área de Design Educacional (DE) pode ser necessário e suficiente para gerar conhecimento aplicável no desenvolvimento de habilidades, competências, atitudes e valores.

O DE surge na Segunda Guerra Mundial, onde um "grande número de psicólogos foi chamado pelo exército dos Estados Unidos da América (EUA) para realizar pesquisas e desenvolver materiais de treinamento com fins militares" (Peixoto et al. 2013). À época era chamado de Design Instrucional, expressão ainda utilizada em diversas partes do mundo, mas já superada no contexto nacional atual. O PROEDU adota o conceito de DE definido por Alves (Alves 2016). Em outras palavras, o DE pode ser compreendido através da noção de "Engenharia Pedagógica" (Silva et al. 2013). O DE possui diversos métodos que podem ser utilizados para "identificar, tipificar e resolver problemas de desempenho" (Oliveira et al. 2015). Um dos métodos amplamente utilizados denomina-se Instructional System Design (ISD) ou Modelo ADDIE. ADDIE é a sigla que se compõem dos seguintes elementos: Analysis, Design, Development, Implementation e Evaluation.

A Figura 1 expressa visualmente as fases: (a) Concepção e (b) Execução; e as etapas (i) Análise, (ii) Desenho, (iii) Desenvolvimento, (iv) Implementação e (v) Avaliação dos projetos educacionais desenvolvidos no âmbito do PROEDU, bem como os produtos gerados ao término de cada etapa.

A solução proposta para o PROEDU, ora denominado de gerador de projetos educacionais, atua fortemente nas duas primeiras etapas: análise e desenho. Na etapa de Análise, o gerador de projetos educacionais conduz o usuário através de um banco de questões e, na etapa de desenho, auxilia a estruturação da solução de aprendizagem organizando os dados e sugerindo alternativas para a implementação. Na sequência, será apresentado o embasamento das análises e o formato de solução proposto pelo sistema.

\section{Análise e desenho da solução}

O modelo ADDIE prevê, a partir de uma demanda de aprendizagem identificada, três etapas: (i) análise do contexto, (ii) análise do público-alvo e (iii) análise dos objetivos 


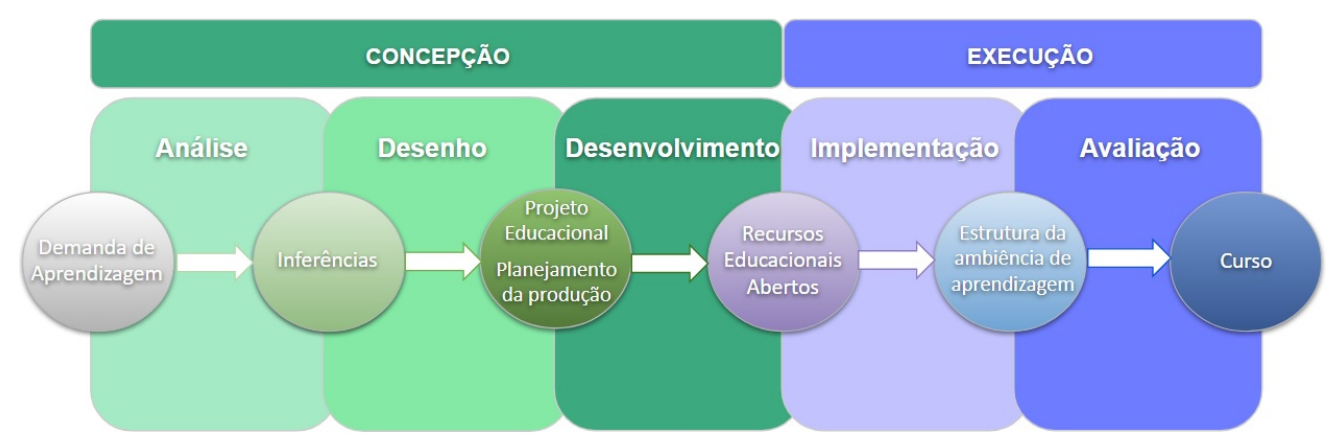

Figura 1. llustração do modelo ADDIE utilizado nos projetos do PROEDU

Fonte: desenvolvido pelos autores

de aprendizagem. A análise do contexto leva em conta os seguintes elementos: infraestrutura tecnológica, verbas disponíveis, restrições, prazos e políticas organizacionais. Já a análise do público-alvo observa as seguintes características dos estudantes: cognitivas, fisiológicas, afetivas e sociais. Os elementos e características servem como ponto de partida para formulação de um banco de questões norteadoras. O usuário é conduzido pelas questões garantindo que informações importantes sejam evidenciadas e levadas em consideração na solução. A análise dos objetivos de aprendizagem é feita em três momentos: (i) formulação do objetivo geral baseada no modelo ABCD (Audiência - Comportamento - Condições - Avaliação), (ii) identificação dos objetivos específicos e (iii) mapeamento dos tipos de aprendizagem envolvidos. O mapeamento dos tipos de aprendizagem tem base na Teoria de Gagné (Gagné 1971).

As análises propiciam um conjunto de inferências que atuam como norteadoras no desenho da solução, o qual contempla os seguintes elementos: (i) formato da solução, local onde se dará o processo de ensino-aprendizagem, (ii) metodologia de ensino, (iii) pré-requisitos tecnológicos, (iv) pré-requisitos de entrada, (v) material de apoio e (vi) matriz de design educacional.

Tais elementos são recomendados pelo gerador de projetos educacionais, a partir dos dados organizados no conjunto de inferências, ou seja, o conjunto de deduções geradas a partir das análises, e são apresentados ao usuário para avaliação e validação. Entre esses elementos, destaca-se a função da matriz de DE, ilustrada pela Figura 2 no modelo empregado pelo PROEDU, pois esta configura-se como um guia das etapas seguintes, quais sejam o desenvolvimento dos recursos educacionais e a implementação da ambiência de aprendizagem. Pode-se observar o caráter organizacional da matriz, pois ela centraliza os objetivos, os tipos de aprendizagem envolvidas no processo e ainda lista todos os recursos que serão produzidos, ou adaptados, aliados às atividades e momentos de avaliação aos quais os estudantes serão submetidos.

\section{Gerador de Projetos Educacionais}

Retomando o objetivo geral apresentado, pode-se afirmar que o gerador de projetos educacionais se propõem a apoiar os núcleos de design educacional que produzem conteúdo educacional, presentes na Rede de Institutos Federais, no desenvolvimento de PE. Lembrando que estes PE servem como norteadores do planejamento educacional de recursos educacionais, métodos e estratégias de ensino, e também conservando a memória da 


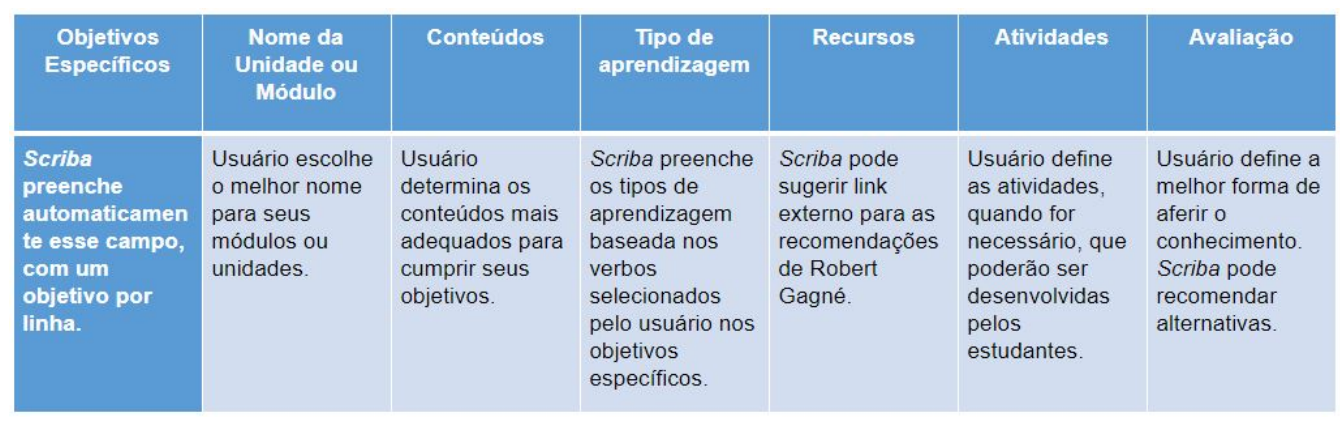

Figura 2. Modelo de matriz de DE adotado no PROEDU

Fonte: desenvolvido pelos autores

produção para futuras adaptações por outros grupos ou instituições. O conjunto de recursos, métodos e estratégias de ensino desenvolvidos serão, posteriormente, adicionados à base de dados do repositório PROEDU, observando-se a taxionomia e as políticas de uso propostas. Nesta perspectiva, o gerador de projetos educacionais visa a implementar o modelo ADDIE 1, no conjunto de etapas incorporadas ao sistema.

\subsection{Design da Aplicação}

As demandas para a composição de recursos educacionais, métodos e estratégias de ensino geradas no âmbito da Educação Profissional e Tecnológica, junto ao objetivo de que estes produtos sejam somados à base do PROEDU fomentaram a concepção da ferramenta de geração de projetos educacionais. Alguns momentos podem ser apontados na concretização do desenho da solução. Primeiro, ter-se o processo de composição de projetos educacionais em um ambiente virtual. Segundo, transpor as principais habilidades do profissional de DE em um modelo de processo para a geração deste documento, o que é dado pelo modelo ADDIE empregado. Terceiro, uma ferramenta com alto nível de usabilidade, contemplar termos de acessibilidade web e agregar componentes inteligentes que auxiliem o profissional, ou grupo, a desenvolver o projeto com qualidade e facilidade. Quarto, colher requisitos para o planejamento junto aos especialistas, como designer web, de experiência de usuário, designer educacional e desenvolvedores. Quinto, a busca por referências em outros modelos de geração de planos de atividades e ferramentas com distribuição dos conceitos elencáveis em classes. Nessa perspectiva, mostra-se na Figura 3 o desenho inicial concebido após um processo de entrevistas baseado no Método Short Bridge (Zilse et al. 2016) para implementar-se o conjunto de funcionalidades e coleta de informações no desenvolvimento da ferramenta.

O design apresentado sucede de diferentes inspirações, passando pelo modelo de design de atividades criativas, do MIT Media Lab, e pelo modo de disposição das informações do modelo de negócios Canvas ${ }^{4}$. O objetivo é ter-se as questões norteadoras inseridas em cada seção, possibilitando uma visão ampla do "todo", associado à granularidade fina de detalhes colhidos do usuário para compor o PE, porém, com grau de liberdade, inspiração e criatividade para inovar. Lembrando que o modelo apresentado representa apenas um esboço da ideia inicial para o desenho da aplicação, ocultadas informações textuais e funcionalidades.

\footnotetext{
${ }^{4}$ Business Model Canvas é uma ferramenta de gerenciamento estratégico
} 


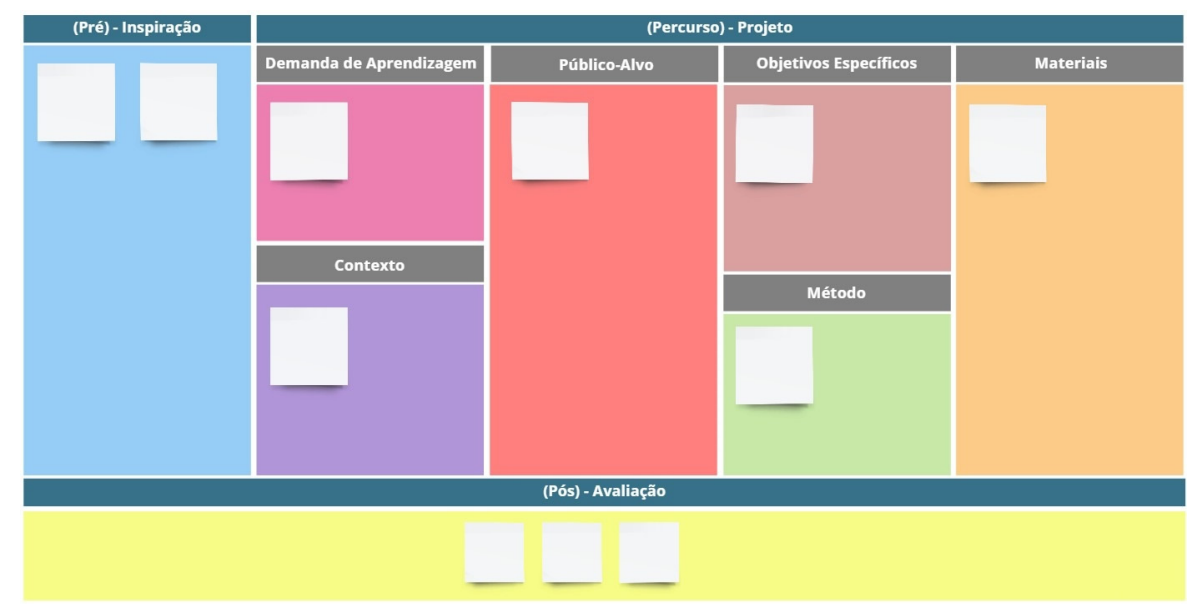

Figura 3. Proposta inicial de design para a ferramenta de geração de projetos educacionais

Fonte: desenvolvido pelos autores

\subsection{Componentes tecnológicos}

O desenvolvimento da solução conta com componentes tecnológicos de armazenamento, processamento, recomendação e recuperação de dados, os quais se relacionam conforme mostra a Figura 4, os quais estão em desenvolvimento com base nas informações detalhadas nas seções 4 e 5.1 .

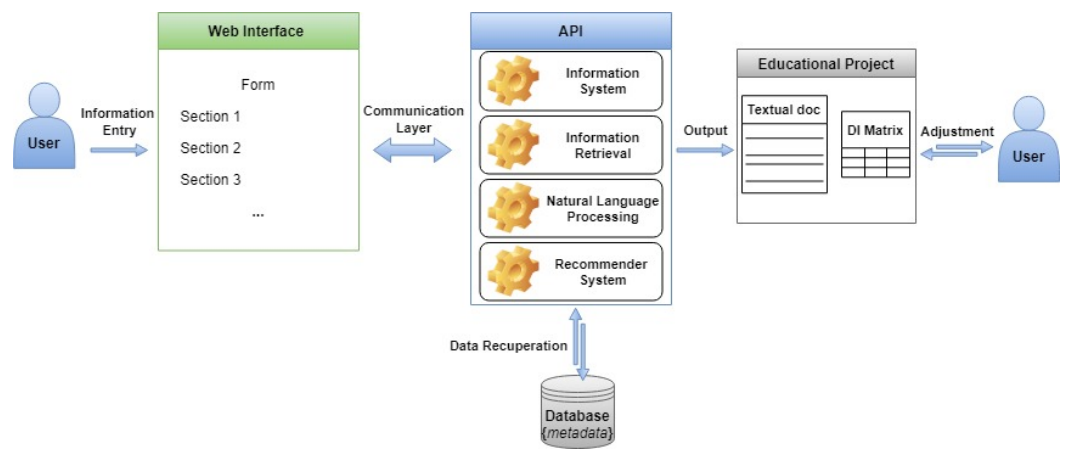

Figura 4. Diagrama de tecnologias da ferramenta de geração de projetos educacionais

Fonte: desenvolvido pelos autores

Quatro principais componentes são previstos: (i) interface web, a qual receberá a entrada de informações do usuário, (ii) interface de aplicações, responsável por tratar e processar informações, (iii) base de dados, onde são armazenadas as informações e (iv) projeto educacional, configurando o produto do processamento das informações na ferramenta. Cada um destes macro-componentes será descrito em maiores detalhes logo em seguida.

A Interface Web é o componente de front-end responsável pela interação direta com o usuário, recebendo a entrada de informações (contemplando tipos textuais, numéricos e array - com o upload de arquivos) em seções individuais correspondentes às informações necessárias previstas com base no modelo ADDIE por meio de um sistema de "pergunta-resposta" ao longo de oito classes: inspiração, demanda, contexto, 
público-alvo, objetivos-específicos, metodologia, materiais e avaliação. Filosoficamente, a interface deve transpor ao usuário a especialidade do profissional de DE, colhendo todas as informações pertinentes à composição do PE em um nível de granularidade fina. Percebe-se, de antemão, o desafio de apresentar terminologias para toda e qualquer área do conhecimento para as quais a ferramenta pode ser utilizada no processo de planejamento e apresentação de soluções de aprendizagem.

Sendo o PE, o produto do processo percorrido na interface e com o objetivo de entregá-lo com a melhor formatação possível, o Sistema de Informação na ferramenta de geração de projetos educacionais conta com diferentes campos tecnológicos aplicados, exigindo o mínimo de edições pelo usuário. Para isso, contamos com componentes específicos, especificados por módulos. Temos nos módulos a rotina promotora de serviços a serem consumidos pelas demais componentes que se beneficiam destes serviços para oferecer suas funcionalidades em uma aplicação. Dizemos que esta interface faz parte do back-end da ferramenta. Dentro dela, estão localizados todas as rotinas para o armazenamento, processamento e recuperação de dados:

- o módulo Recuperação de Informação (RI) tem função justamente de resgatar tanto informações armazenadas na própria ferramenta (como por exemplo, acesso ao PE a qualquer momento) quanto informações externas, como é o caso da possibilidade de upload para documentos de outras bases (como a reutilização de objetos educacionais do próprio repositório PROEDU para referência complementar, por exemplo);

- a Base de Dados (BD) da ferramenta é destinada ao armazenamento das informações referentes ao PE construído utilizando a ferramenta de geração de projetos educacionais. O PE armazenado respeita um padrão de metadados associado, o qual preserva sua estrutura e permite a recuperação e reuso, tendo em vista a memória do ciclo de vida dos produtos técnico-científicos, possibilitando seu acesso atemporal, associado ao login desenvolvedor do PE em questão;

- o emprego de Processamento de Linguagem Natural (PLN) possui relevância tendo em vista que toda informação de entrada e também de saída é no formato de texto em linguagem natural. Algumas das aplicações para PLN são elencadas conforme: indexação e pesquisa em textos longos, recuperação de informação, classificação textual em categorias, extração de informações, tradução automática, resumo automático, pergunta e resposta, extração de conhecimento, geração de textos e diálogos (Chowdhary 2020). Visto que as entradas em cada seção do processo de construção do PE é dado por resposta a uma "questão-norteadora", essa informação necessitará de processamento para a composição do produto do processo, o próprio PE resultante. Dado o objetivo de generalização do uso da ferramenta para as mais diversas áreas de conhecimento, a formatação para a composição de um texto com alto nível de polidez se faz necessária. É também objetivo despender a menor necessidade de ajuste manual, reduzindo a carga de trabalho do usuário. Nesse sentido, vários desafios devem ser enfrentados para alcançar o o resultado esperado, tais como os elencados em (Chowdhary 2020);

- um Sistema de Recomendação (SR) é desejado atuando na ferramenta como componente que auxilia o usuário no correto preenchimento das informações requisitadas para a composição do PE. Basicamente, um SR atua sugerindo itens de forma proativa ao usuário visando complementar ou auxiliar na escolha de itens 
dentro de sistemas que sofrem de sobrecarga de informações. De acordo com (Mobasher et al. 2007), a área de SR pode ser dividida em quatro classificações de acordo com a seguinte taxonomia: (i) baseada em conteúdo; (ii) filtragem colaborativa; (iii) demográfica e (iv) baseada em conhecimento. No primeiro momento, a ferramenta deve contar com uma forma de recomendação menos rebuscada, ou seja, deve realizar recomendações como mecanismo estático auxiliar no modo de "sugestão de escrita" presente em cada campo de preenchimento. A evolução prevista em projeto para este módulo abre espaço para a exploração abrangendo campos como Web Semântica e Ontologias no intuito de aprimorar ações de recomendação, conforme explorado em (Primo et al. 2013; Koster et al. 2015; Behr et al. 2016).

O Projeto Educacional é de fato o produto do processo após as etapas percorridas e o processamento realizado pela ferramenta (output). Assim como as informações de entrada, o PE também é no formato de texto (pode-se perceber a validade do uso de PLN), esse texto possui uma estrutura definida, organizado em seções e subseções. Almejase também a apresentação de uma matriz de DE (tal como apresentado na Figura 2), sintetizando as informações e classificando-as de acordo com o modelo desenvolvido. Tanto a geração do produto textual do PE, quanto da matriz é permitida a intervenção do usuário a fim de realizar alterações, assim como é permitido o retorno à interface de entrada de informações. Tal possibilidade proporciona liberdade ao usuário para editar o projeto, tornando a ferramenta de geração de projetos educacionais uma ferramenta auxiliar no desenvolvimento dos PE e otimizando o tempo de trabalho dos núcleos de produção de conteúdo educacional.

\section{Considerações Finais}

O PROEDU, Repositório de Recursos Educacionais Abertos, foi concebido com o intuito de armazenar e dar acesso público aos recursos educacionais da com possibilidade de reuso e adaptação para outros contextos. Para que a adaptação do conteúdo seja coerente com o planejamento do produto original, faz-se necessário conhecer o PE e que este possa servir de base para as novas versões remixadas do recurso educacional.

Nesse sentido, conhecendo-se os métodos e estratégias contidos no PE, há, supostamente, maior probabilidade de se manter a eficiência nos processos de aprendizagem levando-se em consideração as análises de contexto, público-alvo e os objetivos já realizados na produção de origem do remix. Para isso, o compartilhamento do planejamento, dos métodos e estratégias de ensino implantadas nos recursos educacionais é parte fundamental do processo.

O planejamento educacional com base em metodologias consolidadas fortalece a geração de conhecimento, habilidades, competências, atitudes e valores. Assim sendo, a proposta de desenvolvimento de uma ferramenta de apoio ao desenvolvimento de PE está ancorada nas capacidades expressas pelo DE e baseado no modelo ADDIE. Neste modelo, a ferramenta de geração de projetos educacionais atua diretamente nas etapas de análise e desenho, conduzindo a estruturação da solução de aprendizagem por meio de um conjunto de questões norteadoras dispostas em oito classes distintas (demanda, contexto, públicoalvo, objetivos-específicos, metodologia, materiais, avaliação e referências/inspiração), no intuito de organizar e extrair o máximo de informações para a proposta da solução e armazenamento da memória intelectual da produção. 
A ferramenta encontra-se em fase de desenvolvimento. Entretanto, em seu projeto, componentes tecnológicos são previstos para o armazenamento, processamento e recuperação de dados. Prevê-se o emprego de tecnologias de Processamento de Linguagem Natural e Sistema de Recomendação para auxiliar o usuário a compor o PE para estes objetos com maior qualidade e facilidade. O Projeto Educacional, estruturado por métodos no formato de texto e sintetizado na matriz de DE, compreende o produto do processo compreendido pela ferramenta de geração de PE. Por fim, o produto desta fase de desenvolvimento, quando finalizado, deve ser adicionado ao repositório PROEDU e disponibilizados em conjunto com o Recurso Educacional fruto deste PE.

\section{Referências}

[Alves 2016] Alves, F. (2016). Design de aprendizagem com uso de canvas. DVS Editora.

[Behr et al. 2016] Behr, A., Primo, T., and Viccari, R. (2016). Towards educational metadata interoperability on semantic web. In Brazilian Symposium on Computers in Education (Simpósio Brasileiro de Informática na Educação-SBIE), volume 27, page 1026.

[Chowdhary 2020] Chowdhary, K. (2020). Fundamentals of Artificial Intelligence. Springer.

[Gagné 1971] Gagné, R. M. (1971). Como se realiza a aprendizagem. S.A.

[Koster et al. 2015] Koster, A., Primo, T., Koch, F., Oliveira, A., and Chung, H. (2015). Towards an educator-centred digital teaching platform: the ground conditions for a data-driven approach. In 2015 IEEE 15th International Conference on Advanced Learning Technologies, pages 74-75. IEEE.

[Libâneo 1992] Libâneo, J. C. (1992). Didática. Cortez.

[Mobasher et al. 2007] Mobasher, B., Burke, R., Bhaumik, R., and Williams, C. (2007). Toward trustworthy recommender systems: An analysis of attack models and algorithm robustness. ACM Transactions on Internet Technology (TOIT), 7(4):23-es.

[Oliveira et al. 2015] Oliveira, J. M. d., Csik, M., and Marques, P. (2015). Desenho de cursos: introdução ao modelo addie. Escola Nacional de Administração Pública (Enap).

[Peixoto et al. 2013] Peixoto, A. H., Sondermann, D. V. C., and Silva, J. C. (2013). Designer instrucional em foco: instruções e reflexões sobre um novo campo de ensinar e de saber.

[Primo et al. 2013] Primo, T. T., Behr, A., and Vicari, R. M. (2013). A semantic web approach to recommend learning objects. In International Conference on Practical Applications of Agents and Multi-Agent Systems, pages 340-350. Springer.

[Silva et al. 2013] Silva, C. O. C., Vergara-Nunes, E. L., and Vanzin, T. (2013). Desenho instrucional acessível: materiais didáticos com desenho universal para acesso de alunos cegos ao conhecimento escolar. $13^{\circ}$ ERGODESIGN e USIHC.

[Zabala 2015] Zabala, A. (2015). A prática educativa: como ensinar. Penso Editora.

[Zilse et al. 2016] Zilse, R., Primo, T. T., Koch, F. L., and Koster, A. (2016). An analysis of applying the short bridge method to digital education. In Zaphiris, P. and Ioannou, A., editors, HCI (23), volume 9753 of Lecture Notes in Computer Science, pages 94 102. Springer. 